\title{
Detection of surface defects in logs using point cloud data and deep learning
}

\author{
Shengbo Liu ${ }^{1,2}$, Pengyuan $\mathrm{Fu}^{1}$, Lei Yan ${ }^{1}$, Jian $\mathrm{Wu}^{1,3}$, Yandong Zhao ${ }^{1,3^{*}}$ \\ ${ }^{1}$ School of Technology, Beijing Forestry University, Beijing 100083, China \\ ${ }^{2}$ Beijing Laboratory of Urban and Rural Ecological Environment, Beijing 100083, China \\ ${ }^{3}$ Center for Intelligent Forestry Research, Beijing Forestry University, Beijing 100083, \\ China
}

Received: October 11, 2020. Revised: May 20, 2021. Accepted: July 14, 2021. Published: July 19, 2021.

\begin{abstract}
Deep learning classification based on 3D point clouds has gained considerable research interest in recent years.The classification and quantitative analysis of wood defects are of great significance to the wood processing industry. In order to solve the problems of slow processing and low robustness of 3D data. This paper proposes an improvement based on littlepoint CNN lightweight deep learning network, adding BN layer. And based on the data set made by ourselves, the test is carried out. The new network bnlittlepoint CNN has been improved in speed and recognition rate.
\end{abstract}

The correct rate of recognition for non defect $\log$, non defect log and defect log as well as defect knot and dead knot can reach 95.6\%.Finally, the "dead knot" and "loose knot" are quantitatively analyzed based on the "integral" idea, and the volume and surface area of the defect are obtained to a certain extent,the error is not more than $1.5 \%$ and the defect surface reconstruction is completed based on the triangulation idea.

Keywords: Log defects, Point cloud, Three-dimensional reconstruction, Deep learning, BNLittlePointCNN

\section{INTRODUCTION}

WING to advances in computer technology, sed on 2D views have been proposed. [1].proposed an object recognition framework for threedimensional scenes using the rotation image method. A parameterized feature space algorithm for recognizing the appearance of 3D objects was proposed; this algorithm yielded more accurate results than previous approaches [2],[3].proposed a recognition algorithm based on regular moment invariants. This algorithm considers the regular moment invariant of a target model as an eigenvector and employs a back propagation(BP) neural network to read this eigenvector and achieve the recognition of 3D objects. Subsequently, the development of depth cameras led to further advances.[3] proposed a 3D target recognition method involving the extraction of HoG features from a depth map; however, the results of this method were not ideal owing to the loss of edge information.[4] combined a convolutional neural network and a support vector machine to extract the color image and depth image, respectively, which were then input to a SVM classifier for the classification of objects; this method exhibited hig. 
Recognition accuracy.For instance, to represent point clouds, [5].applied deep learning to $3 \mathrm{D}$ objects represented by point clouds and designed a unified framework of object classification, partial segmentation, and scene semantic segmentation, which was termed Point Net. They succeeded in addressing issues pertaining to point cloud disorder and rotation translation, while achieving a more ideal recognition accuracy. Furthermore,[6] introduced a hierarchical neural network, Point $\mathrm{Net}++$, and achieved a significantly improved classification accuracy by measuring the distance of space and learning local features through the addition of context scales.In addition, domestic scholars have made significant progress in point cloud deep learning.Li Yangyan of Shandong University and others put forward PointCnn based on $\mathrm{x}$ transform matrix, which has broken many world records. The core idea of this method is to sort out the unordered point cloud through an $\mathrm{x}$-transform matrix, and then classify and divide it by using convolutional neural network [8]. However, the disadvantage of this method is that it does not take into account the possibility that the central point is also needed The problem of sorting.A lightweight real-time point cloud network, LightPointNet, is proposed by Bai Jing and $\mathrm{Si}$ Qinglong. The network structure is compact and contains only three layers of convolution, one layer of pooling and one layer of full connection, Moreover, the number of parameters is less than $0.07 \mathrm{~m}$, which greatly simplifies PointNet. In theory, the lightweight feature also determines that the network has better real-time performance. Finally, the author tested the algorithm. It only takes 46 minutes to train modelnet40 with GTX 1080 video card, and only 2.8 MS to identify a single point cloud model, which proves the advantage of the structure framework in real-time performance [9].
The research on $3 \mathrm{D}$ reconstruction technology has also developed rapidly.For the 3D reconstruction of logs, Johan Holmgren et al. used high-density airborne laser scanner data to detect individual trees, segmented the characteristics of individual trees through point clouds, and performed species classification [14]. Michael Thies et al. proposed A method and algorithm for reconstructing the three-dimensional surface of the stem based on the ground laser scanner data of standing trees, composed of overlapping continuous cylinders obtained the tree on the ground by using the portable ground scanning LIDAR IMAGER 5003 The topological map of Rüdiger Hildebrandt and others used a databasedriven method combined with the point cloud data obtained by scanning to perform a threedimensional reconstruction and calculate the volume of a single tree stem. Finally, the test found that the result of the algorithm is different from the result obtained by manual segmentation Less than 1\% [18]. proposed a dual-neighborhood map to reduce the occlusion problem in the point cloud to complete a higher-precision 3D reconstruction [23]. Bournez F et al. evaluated their terrestrial laser scanner (TLS) Data reconstruction algorithm for trees and the other two algorithms PlantScan3D and SimpleTree- the ability to reconstruct the 3D structure of the tree, which can achieve very satisfactory results for the $3 \mathrm{D}$ reconstruction of tree topology and tree volume. In addition, The author also demonstrated that the method has a good performance in reflecting the complexity of branches [26].

Research shows that target recognition approaches based on 3D data have been developed in stages. To address the limitations associated with point cloud data and the complexity of existing networks, a novel lightweight classification network employing 3D vision and deep learning technologies is 
presented herein. Quantitative analysis of the extracted defects, and the results and classification accuracy achieved using this method are analyzed. The results of this study are expected to serve as a technical basis for the efficient and intelligent development of logs.

\section{Materials AND Methods}

\section{A. Data acquisition}

In this study, surface point cloud data of logs are obtained by scanning object surfaces using laser scanning, achieved using Livox-Mid40. This LiDAR(Livox-Mid40) sensor is applicable in various scenarios, such as in mobile robots, distribution robots, security, mapping, and vehicle-road coordination systems. Its maximum detection distance is $260 \mathrm{~m}$ and accuracy is \pm 2 $\mathrm{cm}$. Fig. 1 provides a schematic of the experimental setup.

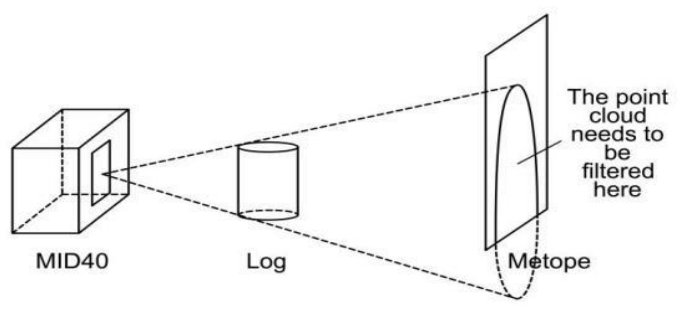

Fig. 1 Experimental setup

\section{B. Specific operation}

Livox-Mid40 is placed in front of the $\log$, as shown in Fig. 1, and the final sampling area obtained is conical. It should be noted that the radar(Livox-Mid40) should be aligned with the axis of the log.In the experiment, the log was placed at a certain distance from the background with the wall as the background. The statistical filtering method is used, and the appropriate number of adjacent points and the threshold value of outlier are set. In order to complete the point cloud filtering task. The log is scanned, and after each scan, the $\log$ is rotated $60^{\circ}$ in the same direction; this process is repeated six times. The data thus obtained are transmitted to a computer for processing. For defect classification, the input data are considered in the form of a matrix; after the extraction of defects, the coordinates of each point cloud are entered into a text file. Thus, we obtained 161 model data.Among these, 116 data were randomly selected as the training dataset, and the remaining 45 data were used as the test dataset. The training dataset included 70 slip knot models and 56 dead knot models; in contrast, the test dataset contained $22 \mathrm{slip}$ knot models and 23 dead knot models.

\section{System construction}

The technical route of this article is divided into five parts: $\log$ data collection, $\log 3 \mathrm{D}$ reconstruction, defective log judgment, log defect identification, $\log$ defect $3 \mathrm{D}$ reconstruction and quantitative analysis. After we use radar to complete the data collection, we first realize the reconstruction of the point cloud $3 \mathrm{D}$ model through the steps of point cloud denoising, registration, and greedy triangulation 3D reconstruction, and then input the point cloud data into BNLightPointCnn for training and testing, and finally judge Whether the log is a defective $\log$, if it is a defective $\log$, input the defect location and its ring point cloud into BNLightPointCnn through threshold screening, then judge the type of log defect, and finally select the defect according to the threshold screened point cloud Extract it for threedimensional reconstruction, and then treat the defect as an irregular object composed of multiple triangular pyramids to calculate the volume and surface area of the defect.Using deep learning technology for defect classification, the recognition effect is better. However, during reconstruction, the smoothness of the generated object is difficult to guarantee strictly by this method. The technology roadmap for this approach is given in Fig.2. 


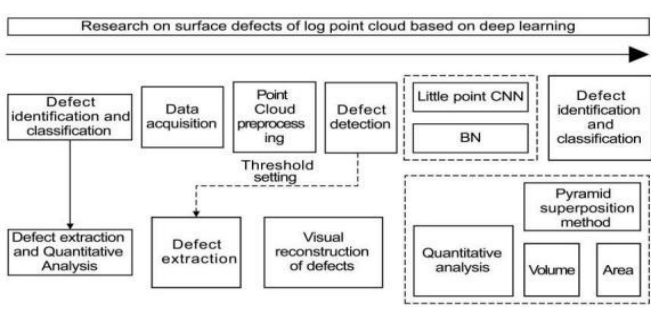

Fig. 2 Technical roadmap

\section{BNLittlePointCNN}

To solve the problem of point cloud disorders, a lightweight convolutional neural network with a $1 \times 3$ convolution core is used to "filter" the convolution layer. The movement of the convolution core is in a $3 \mathrm{D}$ point each time. This imparts a degree of certainty to the input of the convolutional neural network. The convolutional neural network structure is given in Fig.3.It can be seen in the figure, The $\mathrm{n}$ point clouds are input with each point cloud coordinate as a row vector; the next are three convolutional layers, the number of channels of the first convolutional layer is 64 , and the size of the convolution kernel is $1 * 3$; the second volume The number of channels in the accumulation layer is 128 , and the size of the convolution kernel is $1 * 1$; the number of channels in the third convolution layer is 128 , and the size of the convolution kernel is $1 * 1$; then the data is input to the pooling layer for maximum pooling processing, Each channel is subjected to maximum pooling to obtain a 128-dimensional vector; then a $1 * 256$ vector is obtained through the fully connected layer. In order to prevent overfitting, a Dropout with a dropout rate of 0.5 is added after the fully connected layer; Then get $\mathrm{k}$-dimensional output, where $\mathrm{k}$ is the number of categories. To improve the accuracy and speed of the network operation, we improved LittlePointCNN and developed BNLittlePointCNN. In this study, a BN(batch normalization) layer is added to ensure the selection of appropriate learning rate, parameter initialization, weight attenuation coefficient, and drop out ratio, as well as to improve the convergence rate of the algorithm and control over-fitting. In LittlePointCNN, the network is normalized through the addition of a $\mathrm{BN}$ layer before every layer, except the fully connected layer, and the network is simplified by eliminating the dropout layer. The BNLittlePointCNN network architecture is shown in Fig. 4.

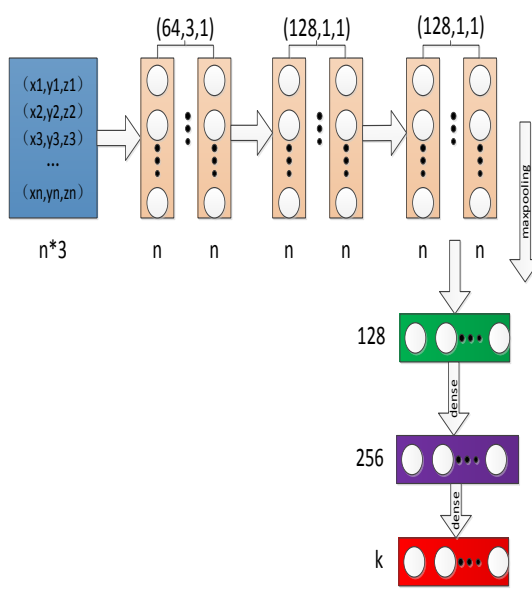

Fig. 3 LittlePointCNN architecture

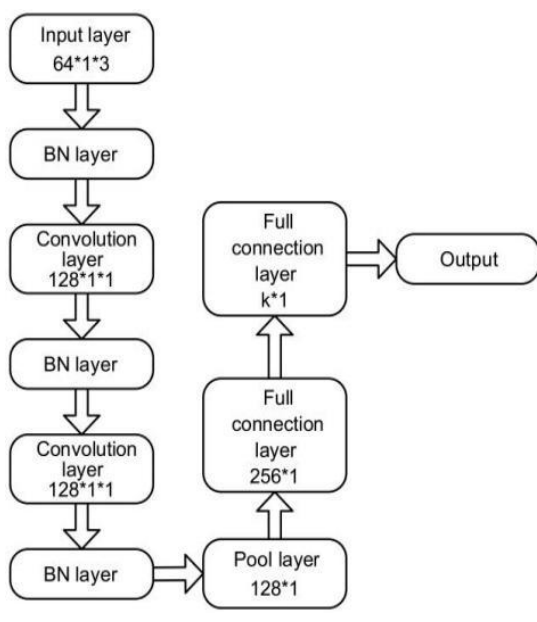

Fig. 4 BNLittlePointCNN architecture

\section{E. Log defect recognition using}

\section{BNLittlePointCNN}

The point cloud data were identified and classified using BNLittlePointCNN, and the results thus obtained were categorized as non-log, defective logs, and non-defective logs. If the classification result indicates a defective log, By calculating the average radius of the log to determine the threshold required to extract the 
defect. Subsequently, the extracted point cloud data are input to the network in order to identify defects. After the point cloud data are assessed using BNLittlePointCNN1, the log data containing defects are input to the defect extraction part.In the defect extraction section.By setting a suitable threshold value, the defects in the log can be extracted, and the maximum and minimum values of the defects on the log length axis are calculated. Extracts the coordinates of all the points that fall within the axis of the log point cloud's length direction and 5,000 points from these points as input to the convolutional neural network. The flowchart of the defect detection and extraction process is presented in Fig. 5.

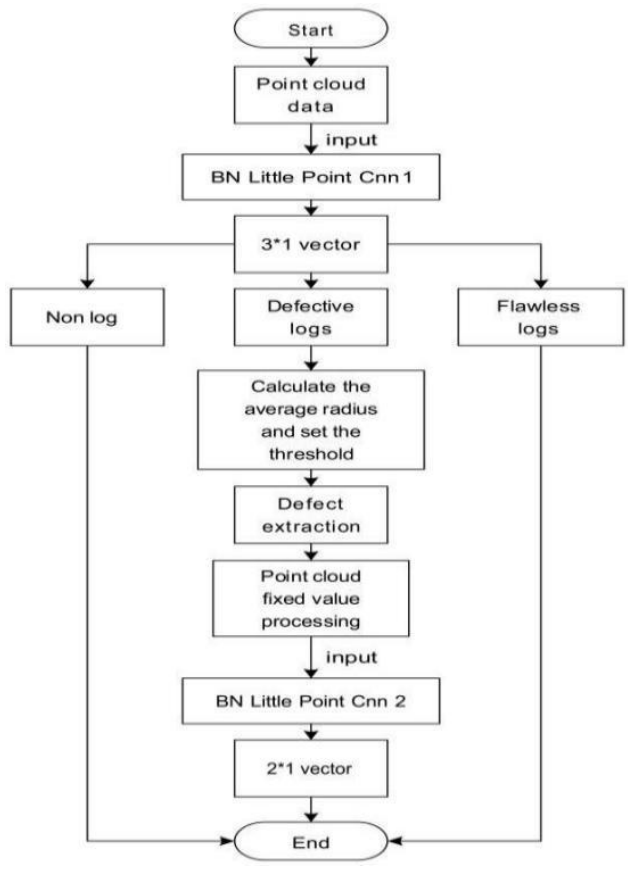

Fig. 5 Flowchart for log defect detection and extraction

\section{F. Quantitative Analyses}

After the extraction of defects, the volume of these defects is calculated by dividing the volume of the entire point cloud data into the volumes of several triangular pyramids. For close approximation to actual values, the overall defect needs to be divided into several triangular cones; this process is illustrated in Fig. 6.

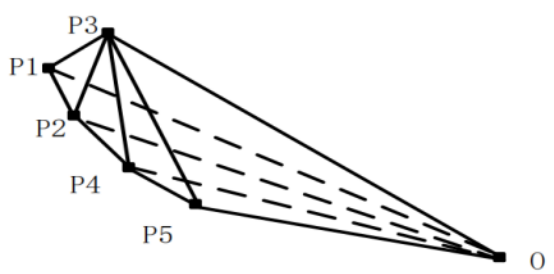

Fig. 6 Schematic of the triangular cones method

The order is based on the coordinate size of each point $\mathrm{p}$ on the horizontal axis. The volumes of triangular cones O-P1P2P3, O-P2P3P4, and OP3P4P5 are used to calculate the total volume.For the calculation of defect surface area, we adopt the same method as volume calculation. The flowcharts of the two programs are depicted in Fig. 7 and Fig.8.

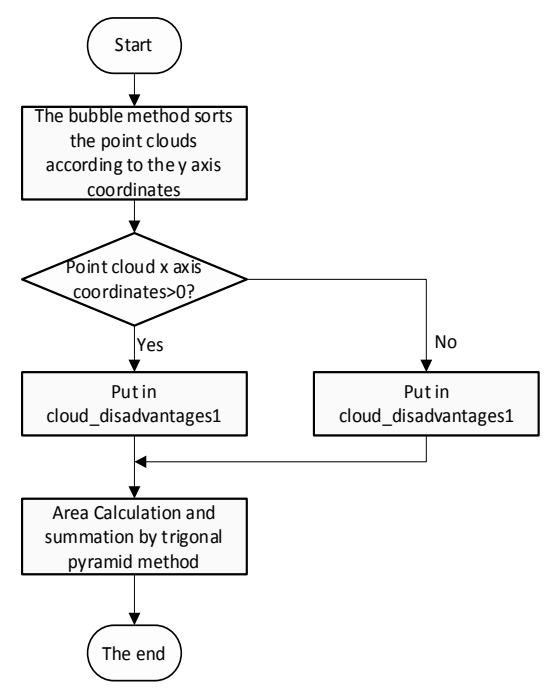

Fig. 7 Flowcharts of programs used to calculate volume and surface area

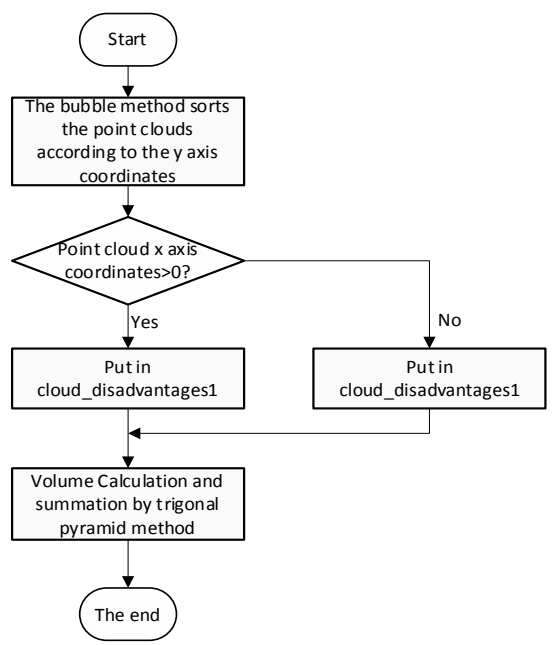

Fig. 8 Flowcharts of programs used to calculate volume and surface area 


\section{Results}

To evaluate the effectiveness of the defect recognition performance of the proposed method, the acquired datasets were used to conduct classification and quantitative analyses. From a total of 161 model data, 116 data were randomly selected as the training dataset, while the remaining 45 data were used as the test dataset. The training dataset included 70 slip knot models and 56 dead knot models, whereas the testing dataset included 22 slip knot models and 23 dead knot models.
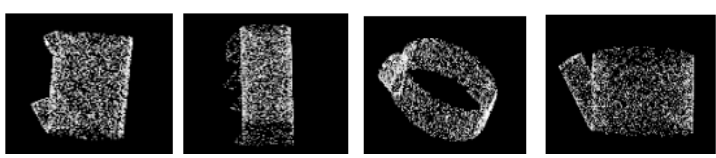

Fig. 9 Input data instances

Correlation Parameters

\section{Activation function}

Commonly used activation functions include the sigmoid, tanh, and ReLu functions. Generally, the tanh and sigmoid functions are deemed suitable when data features are evident and obscured, respectively. However, when using these two activation functions, it is necessary to normalize the input to ensure that expressions of original features are retained. In contrast, the $\mathrm{ReLu}$ function is more ideal; therefore, it was used in this study.

\section{Optimizer}

An optimizer is a parameter used for compilation in the Keras model, which is used to accelerate the training of convolutional neural networks. Commonly used optimizers include the stochastic gradient descent, RMSPROP, Adagrad, Adadelta, Adam, Adamax, and Nadam. To select a suitable optimizer, the same training and test datasets were tested using different optimizers, considering training time and accuracy as the evaluation criteria. The results thus obtained are listed in Table 1.

\begin{tabular}{|c|c|c|}
\hline Optimizer & $\begin{array}{c}\text { Training } \\
\text { time }\end{array}$ & Accuracy \\
\hline SGD & $5 \min 40 \mathrm{~s}$ & $68.9 \%$ \\
\hline RMSPROP & $5 \min 33 \mathrm{~s}$ & $91.1 \%$ \\
\hline Adagrad & $5 \min 53 \mathrm{~s}$ & $88.9 \%$ \\
\hline Adadelta & $5 \min 50 \mathrm{~s}$ & $53.3 \%$ \\
\hline Adam & $5 \min 36 \mathrm{~s}$ & $91.1 \%$ \\
\hline Adamax & $5 \min 43 \mathrm{~s}$ & $84.4 \%$ \\
\hline Nadam & $5 \min 43 \mathrm{~s}$ & $88.9 \%$ \\
\hline
\end{tabular}

Table 1

Training results of different optimizers

The accuracy in the table is the accuracy during the test set test. The accuracy rate is defined by the following formula:

$$
\text { accuracy }=(T P+T N) /(P+N)
$$

Among them, $\mathrm{P}=\mathrm{TP}+\mathrm{FN}$ represents the number of samples that are actually positive,TP:The number of positive examples,FP:The number of positive examples that were incorrectly classified,FN:The number of cases that were incorrectly classified as negative, TN:The number of correctly classified as negative examples.

According to the characteristics of each optimizer algorithm, we know that RMSprop, Adadelta, Adam have similar effects in many cases.From the table, it is evident that all the optimizers require more than $5 \mathrm{~min}$ for training. Overall, RMSPROP exhibited the shortest training time. Furthermore, Adam and RMSPROP were the most accurate optimizers, both exhibiting an accuracy of $91.1 \%$. Thus, in this study, RMSPROP was selected as the optimizer, considering both training time and accuracy.

\section{Loss function}

The loss function reflects the degree of data fitting achieved by a model. For a good fit, the value of the loss function is low, and vice versa. 
The loss function is derived based on the difference between the predicted and actual values; the value of this function is then used to update the weights for the next iteration. This iterative process continues until the model achieves the lowest value for the loss function, which is indicated by negligible or a lack of change in the loss function value; at this instance, the model is considered to have converged. Commonly used loss functions include the mean square error, absolute variance, average absolute percentage error, mean square logarithmic error, logarithmic loss, and various types of logarithmic loss. In this study, the optimal loss function is determined by using different loss functions for the same training and test datasets and by considering training time and accuracy as the evaluation criteria:

\begin{tabular}{|l|l|l|}
\hline Loss function & Loss & $\begin{array}{l}\text { Accurac } \\
\mathrm{y}\end{array}$ \\
\hline (binary_crossentropy) & 0.245 & $91.1 \%$ \\
\hline (categorical_crossentropy) & 0.246 & $91.1 \%$ \\
\hline (cosine_proximity) & 0.460 & $88.9 \%$ \\
\hline (Mse) & 0.077 & $80.0 \%$ \\
\hline (mae) & 0.311 & $88.9 \%$ \\
\hline
\end{tabular}

Table 2

Training results of different loss function

As shown in the table, mean square error exhibits the lowest total loss, while the logarithmic loss function and the logarithmic loss function of various classes exhibit the highest accuracy $(91.1 \%)$. Considering these results, the logarithmic loss function was employed in this study.

\section{Number of iterations}

Based on the results discussed above, experiments involving different epochs were conducted to determine the relationship between the number of iterations and accuracy; the results of these experiments is shown in Fig. 10.

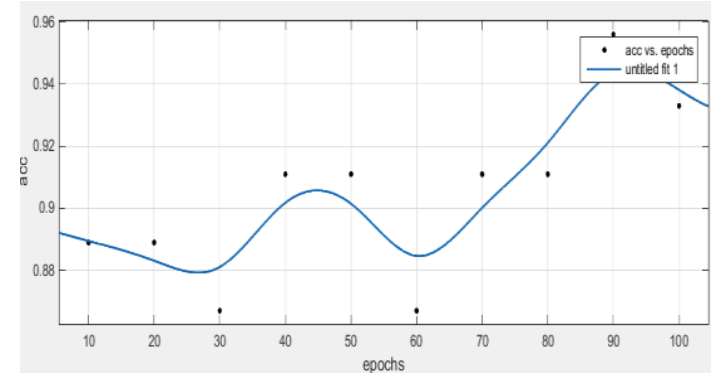

Fig. 10 Training results for different epochs

As can be seen from the above figure, with the increase of iteration times, the accuracy rate basically increases. When epochs is less than 100 , the accuracy rate is $95.6 \%$ when epochs $=90$. Therefore, considering the time cost and accuracy, the iteration number is set to 90 .

\section{Defect identification results}

Using the parameters set for BNLittlePointCNN, we elucidate the relationship between the accuracy of the training and test datasets and the number of iterations; This relationship is shown below on the left.With an increase in the number of iterations, the training accuracy for both the training and the test datasets exceeds 95\%. Using the same parameters and datasets as BNLittlePointCNN, LittlePointCNN is employed for classifying slip knot and dead knot defects, and the results are shown in Fig. 11.
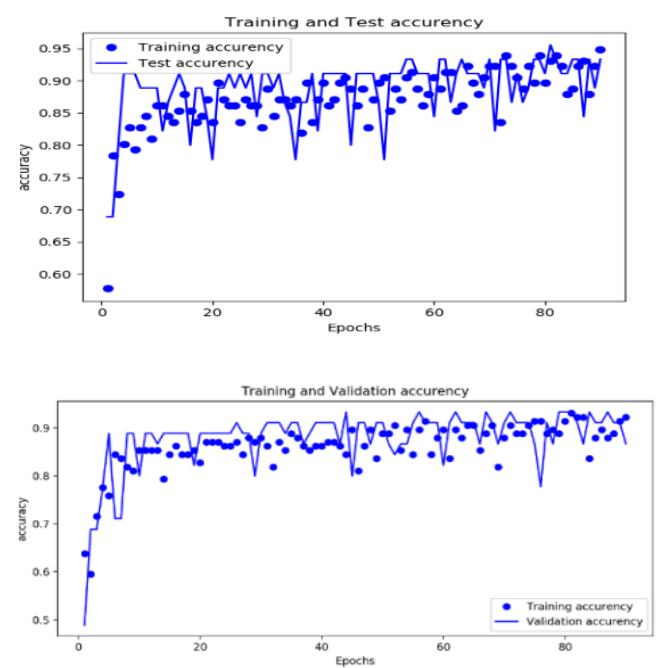

Fig.11Comparison of BNLittlePointCNN and

LittlePointCNN log defect classification training

As shown in Fig. 11, the maximum accuracy for the training and test datasets is $93.3 \%$, which is 
slightly lower than that of BNLittlePointCNN. Results of a comparison between these classification results are presented in Table 3.

\begin{tabular}{|c|c|c|}
\hline Training network & Loss & Accuracy \\
\hline BNLittlePointCNN & 0.195 & $95.6 \%$ \\
\hline LittlePointCNN & 0.245 & $93.3 \%$ \\
\hline
\end{tabular}

Table 3

Comparison result of BNLittlePointCNN and LittlePointCNN

Thus, the loss in BNLittlePointCNN is less than that in LittlePointCNN, and BNLittlePointCNN is more accurate than LittlePointCNN. Thus, BNLittlePointCNN is superior to LittlePointCNN in terms of the training results.

After that, considering the wide applicability of point-Net, the pioneering network of 3D deep learning, the point-Net, pointNet++, and BNLittlePointCNN network test situations were compared with the same data. As shown in the table below.

\begin{tabular}{|l|c|c|}
\hline Training network & Loss & Accuracy \\
\hline BNLittlePointCNN & 0.195 & $95.6 \%$ \\
\hline Point-Net & 0.205 & $94.3 \%$ \\
\hline Point-Net++ & 0.215 & $92.6 \%$ \\
\hline
\end{tabular}

Table 3

Comparison result of BNLittlePointCNN and Point-Net and Point-Net++

From this comparison, it can be seen that from the perspective of classification accuracy, the improved LittlePointCNN is higher than PointNet++ and lower than Point-Net. After adding the BN layer, the classification accuracy is improved, which is higher than the previous two networks.

\section{Extraction and quantitative analyses of $\log$ defects}

Using the previous log point cloud data and the visualization experiments, the following experimental results were obtained.

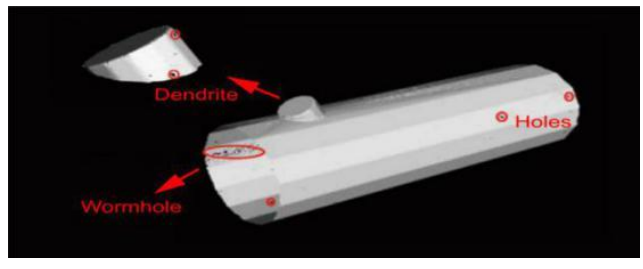

Fig. 12 - 3D reconstruction of log point cloud data

We carried out the above calculation operation to the extracted flaw model. The volume of the $\log$ was determined to be

$$
V^{\prime}=0.486708
$$

The final volume obtained via data conversion is

$$
V=V^{\prime} \times a^{3}=0.487 \times 25^{3}=7609.375 m^{3}
$$

The deviation from the theoretical value is expressed as follows:

$\Delta_{V}=\frac{\left|V-V_{0}\right|}{V_{0}}=\frac{|7609.375-7710.521|}{7710.521}=1.312 \%$

The surface area of the defect can be calculated as follows:

$S=S^{\prime} \times a^{3}=4.166 \times 25^{2}=2603.75 m^{2}$

Thus, the error in the result obtained using this method can be calculated as follows:

$$
\Delta_{S}=\frac{\left|S-S_{0}\right|}{S_{0}}=\frac{|2603.75-2623.29|}{2623.29}=0.745 \%
$$

As can be seen, the error in the final result is less than $1.5 \%$ for both the volume and the surface area. Compared with the basic integral method, this error is low, indicating that the proposed method is more accurate.

\section{CONCLUSIONS}

This paper presented a log defect classification method based on 3D laser scanning and point cloud data. A novel processing network, BNLittlePointCNN, is presented; this network optimizes the Point cloud depth learning network, LittlePointCNN. The BNLittlePointCNN network is constructed by adding a BN layer after each convolution layer. When using this approach, $\log$ defects are extracted via preprocessing of cloud point data and by using a defect recognition algorithm. Furthermore, quantitative analyses of the proposed method are 
conducted using the cumulative pyramid method, based on the idea of "differential."

The proposed BNLittlePointCNN network is used to identify the knot defects in logs, achieving a classification accuracy of $95.6 \%$. Relative to the theoretical values, the error in the results obtained via the proposed method was less than $1.5 \%$.

The research conclusions of this paper can provide technical support for intelligent log processing, identify and quantitatively analyze the defects of logs, and meet the requirements of intelligent and efficient log processing.

\section{REFERENCES}

[1] Date, H., Kaneta, Y., Hatsukaiwa, A., et al. (2012). Object recognition in terrestrial laser scan data using spin images. Computer-Aided Design and Applications, 9(2), 187-197.

[2] Holmgren, J., Persson, A. (2004). Identifying species of individual trees using airborne laser scanner. Remote Sensing of Environment, 90(4), 415-423.

[3] Janoch, A., Karayev, S., Jia, Y., et al. (2012). A category-level 3D object dataset: putting the kinect to work. London: Springer, 141165.

[4] Wang Y, Sun Y, Liu Z, et al. Dynamic Graph CNN for Learning on Point Clouds[J]. 2018.

[5] Qi, C. R., Su, H., Mo, K., et al. (2016). Point Net: Deep learning on point sets for 3D classification and segmentation. ar Xiv preprint ar Xiv:1612.00593, 77-85.

[6] Qi, C. R., Yi, L., Su, H., et al. (2017). Point Net++: Deep Hierarchical Feature Learning on Point Sets in a Metric Space. ar Xiv preprint ar Xiv:1706.02413.

[7] Teobaldelli, M., Puig, A. D., Zenone, T., et al. (2008). Building a topological and geometrical model of poplar tree using portable on-ground scanning LIDAR[J]. Functional Plant Biology, 35(10), 1080.

[8] Tian, X. Z., Wen, J. W., Jun, F. (2006). A novel multi-scale intelligent recursive recognition method for three-dimensional moving targets[J]. Acta Automatica Sinica, 32(5), 641-657.

[9] Zhou, Zhiyong; Dai, Yakang.(2019) An efficient algorithm for defect reconstruction from 3D point cloud.Proceedings of SPIE The International Society for Optical Engineering, v 11069, 2019.

[10]Yu, L. (2010). Wood CT detection and 3D structure reconstruction based on multifractal spectrum technology. Harbin: Northeast Forestry University.

[11]Zhao, P., Zhao, Y., Chen, G. (2017). Quantitative analysis of wood defects based on 3D scanning technology. Journal of Agricultural Engineering, 07, 178-183

[12]Zheng, J. (2013). Research and implementation of single tree measurement method based on 3D laser scanning technology. Beijing Forestry University.

[13]Xu Shanshan, Xu Sheng, Liu ying'an.wood defect recognition based on convolutional neural network [J]. Journal of Shandong University (Engineering Edition), 2012, 43 (2)

[14]Zhang Shaoqun, Hua Jun, Xu Wei, et al. Fractal characteristics of wood fracture based on 3D scanning technology [J]. Forestry science, 2014, 50 (7): 138-142

[15]Guan Xipeng. Research on tree 3D point cloud data analysis and modeling technology [D]. Changsha: Central South University of forestry science and technology, 2015

[16]Yingxue Zhang, Michael Rabbat. A GRAPHCNN FOR 3D POINT CLOUD CLASSIFICATION[C]// ICASSP 2018 2018 IEEE International Conference on Acoustics, Speech and Signal Processing (ICASSP). IEEE, 2018.

[17] Bournez E , Landes T , Saudreau M , et al. FROM TLS POINT CLOUDS TO 3D MODELS OF TREES: A COMPARISON OF EXISTING ALGORITHMS FOR 3D TREE RECONSTRUCTION[J]. ISPRS International Archives of

[18]Rüdiger Hildebrandt, André Iost. From points to numbers: a database-driven approach to convert terrestrial LiDAR point clouds to tree volumes[J]. European Journal of Forest Research, 2012, 131(6):1857-1867.

[19]Méndez, Valeriano, Rosell-Polo J R, Pascual $M$, et al. Multi-tree woody structure reconstruction from mobile terrestrial laser scanner point clouds based on a dual neighbourhood connectivity graph algorithm[J]. Biosystems Engineering, 2016, 148:34-47.

[20]Ye Ning, Wang Houli, Xu Zhaojun, et al. Wood defect recognition based on support vector machine $[\mathrm{J}]$. Computer application and software, 2006, 23 (4)

[21]Li Qiu, Qin Yongzhi, Li Hongying. Application of laser 3D scanning technology in surface subsidence monitoring of mining area [J]. Coal Engineering (4): 98-100

[22]Zhang Jun. application of laser threedimensional scanning technology in existing building survey [C] / / Proceedings of 2013 technical exchange meeting on function improvement of existing buildings. 2013 
[23]Michael, T., Pfeifer, N., Winterhalder, D., et al. (2004). Three-dimensional reconstruction of stems for assessment of taper, sweep and lean based on laser scanning of standing trees. Scandinavian Journal of Forest Research, 19(6), 571-581.

[24]Niu Xiaojing. Research on 3D point cloud processing and reconstruction method of maize plant [D]. Shaanxi: Northwest Agricultural and Forestry University, 2016

[25]Yu Lei, Qi Dawei. Study on detection of wood defects by computer tomography $[\mathrm{J}]$. Forest engineering, 2006, 22 (5): 13-15

[26]Wang zaishan. 3D reconstruction of wood CT images [D]. Harbin: Northeast Forestry University, 2015

[27]Liu Hongwei. Establishment of 3D tree model based on tlidar point cloud data [D]. Beijing Forestry University, 2011

[28]Zheng Jun. research and implementation of single tree measurement method based on 3D laser scanning technology [D]. Beijing Forestry University, 2013

[29]Yao, L., Jie, M., Ji, Z., et al. (2012). Threedimensional automatic target recognition based on spin-images. Infrared and Laser Engineering, 41(2), 543-548.

[30]Sahebdivani, Shima Arefi, Hossein; Maboudi, Mehdi.(2020) Deep Learning based Classification of Color Point Cloud for 3D Reconstruction of Interior Elements of BuildingsIranian Conference on Machine Vision and Image Processing, MVIP, v 2020February

[31]Ashkan Tashk, J?rgen Herp, Esmaeil Nadimi, Automatic Segmentation of Colorectal Polyps based on a Novel and Innovative Convolutional Neural Network Approach, WSEAS Transactions on Systems and Control, ISSN / E-ISSN: 1991-8763 / 22242856, Volume 14, 2019, Art. \#47, pp. 384391.

[32]Aneta Zemankova, Artificial Intelligence and Blockchain in Audit and Accounting: Literature Review, WSEAS Transactions on Business and Economics, ISSN / E-ISSN: 1109-9526 / 2224-2899, Volume 16, 2019, Art. \#64, pp. 568-581.

[33]Hira Lal Gope, Hidekazu Fukai, Normal and Peaberry Coffee Beans Classification from Green Coffee Bean Images Using Convolutional Neural Networks and Support Vector Machine, Engineering World, Volume 2, 2020, pp. 175-182.
Shengbo Liu, born in 1989, received his Master degree in control theory and control engineering from Beijing Forestry University in 2014. Now he works in the internship and experiment center of the school of technology in Beijing Forestry University. He is interested in researching of computer aided automation.

Pengyuan Fu, born in 1997, received his bachelor's degree in engineering from Chengdu University of Information Engineering in 2019. Since then, He has been studying for a master's degree in Beijing Forestry University, majoring in control engineering.

Lei Yan, born in 1979, is a professor at Beijing, Forestry University. He is interested in machine vision and artificial intelligence.

Jian Wu, born in 1989, studied in Beijing Forestry University at 2006 2013, received his master degree in mechanical manufacturing and automation from Beijing Forestry University in 2013. He is interested in researching intelligent control and intelligent detection technology.

Yandong Zhao, born in 1965, is a professor at Beijing Forestry University. She received her Doctor's degree in China Agricultural University. She is interested in intelligent detection and information processing.

\section{Creative Commons Attribution License 4.0 (Attribution 4.0 International, CC BY 4.0)}

This article is published under the terms of the Creative Commons Attribution License 4.0 https://creativecommons.org/licenses/by/4.0/deed.en_US 